\title{
REDUCIBILITY MOD $p$ OF INTEGRAL CLOSED SUBSCHEMES IN PROJECTIVE SPACES - AN APPLICATION OF ARITHMETIC BÉZOUT
}

\author{
REINIE ERNÉ
}

\begin{abstract}
In [4], we showed that we can improve results by Emmy Noether and Alexander Ostrowski ([8]) concerning the reducibility modulo $p$ of absolutely irreducible polynomials with integer coefficients by giving the problem a geometric turn and using an arithmetic Bézout theorem ([2]). This paper is a generalization of [4], where we show that combining the methods of [4] with the theory of Chow forms leads to similar results for integral, closed subschemes of arbitrary codimension in $\mathbf{P}_{\mathrm{Z}}^{s}$.
\end{abstract}

Introduction. Let $K$ be a number field with ring of integers $R$, and $Z$ a flat, integral, closed subscheme of dimension $r+1$ and degree $d$ in $\mathbf{P}_{R}^{s}(s, d \geq 2)$, with absolutely irreducible generic fiber. One can show that the fiber $Z_{k(p)}$ is also absolutely irreducible for all but finitely many prime ideals $p$ of $R$ (e.g., [5, Theorem 9.7.7] and [6, Theorem 4.10]).

We would like to bound the (product of the) norms of the prime ideals $p$ of $R$ for which the fiber $Z_{k(p)}$ is not absolutely irreducible in terms of the projective height of $Z$, as defined in [2]. In this paper, using arithmetic intersection theory, we solve for any fixed $n<d$ the analogous problem obtained by replacing "absolutely irreducible" by "is not a union of two closed subschemes of degrees $n$ and $d-n$, respectively". To prove this theorem, we use Chow forms, and translate the problem to bounding the height of an intersection in some projective space. Thus, the proof becomes a straightforward application of an arithmetic Bézout Theorem for non-proper intersections given in [2], which reduces it to bounding degrees and heights of specific cycles in terms of the data provided.

Thanks. I would like to thank Hervé Billard for asking me about higher codimensions when I gave a talk in Brest on the irreducibility of hypersurfaces. I would also like to thank Teresa De Diego, who took the time during a conference in Obernai to tell me about Chow forms.

Some notation. Given a ring $R$ as above, and a locally free $\mathcal{O}_{\text {Spec }(R)}$-module $\mathcal{E}$ of finite rank $s+1(s \geq 0)$, let $\mathbf{P}(\mathcal{E})=\operatorname{Proj}_{\text {Spec }(R)}\left(\operatorname{Sym}\left(\mathcal{E}^{\vee}\right)\right)$ be the associated space of lines, where $\mathcal{E}^{\vee}$ denotes the dual sheaf of $\mathcal{E}$, and let $\pi$ denote its structural morphism. We suppose $\mathcal{E}$ endowed with a Hermitian metric $h$, and endow $\mathcal{E}^{\vee}$ with the dual metric.

Received March 24, 2000. 
Let $r$ be a positive integer. For $i=0, \ldots, r$, let $\mathbf{P}_{i}=\mathbf{P}(\mathcal{E})$, and $\mathbf{P}_{i}^{\vee}=\mathbf{P}\left(\mathcal{E}^{\vee}\right)$. We endow the canonical quotient line bundle $\mathcal{O}(1)$ on $\mathbf{P}_{i}^{\vee}$ with the quotient metric (cf. $[2,3.1 .2 .3]$ ), and let $\overline{M_{i}}$ be the pullback of the resulting Hermitian line bundle $\overline{\mathcal{O}(1)}$ on $\mathbf{P}_{i}^{\vee}$ to $\prod_{i=o}^{r} \mathbf{P}_{i}^{\vee}$.

Finally, for $x \in \mathbb{N}$, let $F_{x, r}(\mathcal{E}):=\otimes_{i=0}^{r} \operatorname{Sym}^{x}(\mathcal{E})$

Chow divisors and forms. By [2, 4.3.1], we can associate to each non-zero algebraic cycle $Z \in Z_{r+1}(\mathbf{P}(\mathcal{E}))$ a Chow divisor $\mathrm{Ch}_{\mathbf{1}}(Z)$ (where $\mathbf{1}=(1, \ldots, 1) \in$ $\left.\mathbb{Z}^{r+1}\right)$ in $Z^{1}\left(\prod_{i=0}^{r} \mathbf{P}_{i}^{\vee}\right)$, which is effective (resp. flat, resp. flat and irreducible) if such is the case for $Z$.

Let $Z$ now be a non-zero effective cycle of degree $x$ in $Z_{r+1}(\mathbf{P}(\mathcal{E}))$. Generically, the associated Chow divisor $\mathrm{Ch}_{\mathbf{1}}(Z)_{K}$ is the divisor of a non-zero multihomogeneous form $\phi_{\mathbf{1}, Z_{K}}$ in

$$
H^{0}\left(\prod_{i=0}^{r}\left(\mathbf{P}_{i}^{\vee}\right)_{K}, \otimes M_{i, K}^{x}\right) \cong F_{x, r}(\mathcal{E})_{K},
$$

called the Chow form of $Z_{K}$. Thus we can associate a point of $\mathbf{P}\left(F_{x, r}(\mathcal{E})\right)(K)$ to each non-zero effective cycle of degree $x$ in $Z_{r+1}(\mathbf{P}(\mathcal{E}))$. If the class number of $K$ is one, there exists a generalized Chow form $\phi_{\mathbf{1}, Z}$ over $R$, for which $\mathrm{Ch}_{\mathbf{1}}(Z)=$ $\operatorname{div}\left(\phi_{\mathbf{1}, Z}\right)$ in $\prod \mathbf{P}_{i}^{\vee}$. Similarly, for every point $t$ of $\operatorname{Spec}(R)$, we can define Chow divisors and forms for the cycles contained in the fiber above $t([2,4.3 .2])$.

If $Z$ is moreover flat over $\operatorname{Spec}(R)$, we have the following result:

Proposition. Let $Z \in Z_{r+1}(\mathbf{P}(\mathcal{E}))$ be a flat, integral, closed subscheme of $\mathbf{P}(\mathcal{E})$ of degree $x$, with Chow divisor $\mathrm{Ch}_{\mathbf{1}}(Z)$. Let $\phi_{K}$ be the Chow form of $Z_{K}$. Let $\left[\phi_{K}\right] \in \mathbf{P}\left(F_{x, r}(\mathcal{E})\right)(K)$ be the corresponding point, and $P_{Z}$ its Zariski closure in $\mathbf{P}\left(F_{x, r}(\mathcal{E})\right)$. Then for every point $t$ of $\operatorname{Spec}(R)$, the fiber $P_{Z, t}$ is the point of $\mathbf{P}\left(F_{x, r}(\mathcal{E})\right)_{t}$ corresponding to the Chow form $\phi_{t}$ of $Z_{t}$.

Proof. It suffices to note that by construction, we have $\mathrm{Ch}_{\mathbf{1}}\left(Z_{t}\right)=\mathrm{Ch}_{\mathbf{1}}(Z)_{t}$ for every point $t$ of $\operatorname{Spec}(R)([2,4.3 .2])$. In particular, as $Z$ is flat, the Zariski closure of $\operatorname{div}\left(\phi_{K}\right)=\mathrm{Ch}_{\mathbf{1}}\left(Z_{K}\right)$ is $\mathrm{Ch}_{\mathbf{1}}(Z)$.

Components of degree $\mathbf{n}$. Let $d \in \mathbb{N}_{>0}$, and fix integers $1 \leq n \leq d-1$ and $0 \leq r \leq s$. Let us simplify the notation by setting $F_{x}:=F_{x, r}(\mathcal{E})$ for every $x$. Consider the morphism

$$
\psi: \mathbf{P}\left(F_{n}\right) \times \mathbf{P}\left(F_{d-n}\right) \rightarrow \mathbf{P}\left(F_{d}\right)
$$

defined by taking the product on sections (seen as multihomogeneous forms on $\prod \mathbf{P}_{i}^{\vee}$ ), i.e., on sections, $\psi$ corresponds to taking the union of two cycles of degrees $n$ and $d-n$ in $Z_{r+1}(\mathbf{P}(\mathcal{E}))$ in order to obtain one of degree $d$. Let $\mathcal{W}$ denote the image of $\psi$.

Let $Z$ be a flat, integral, closed subscheme of degree $d$ in $Z_{r+1}(\mathbf{P}(\mathcal{E}))$, and let $P_{Z}$ be as in the proposition. By dimension arguments and the proposition, the intersection of $P_{Z}$ and $\mathcal{W}$ is either $P_{Z}$, if $Z_{\bar{K}}$ has a component (irreducible or not) of degree $n$, or a finite number of closed points whose images under the 
structural morphism $\pi: \mathbf{P}\left(F_{d}\right) \rightarrow \operatorname{Spec}(R)$ are the prime ideals $q_{1}, \ldots, q_{v}$ above which the fiber of $Z \rightarrow \operatorname{Spec}(R)$ has such a component.

Before stating the theorem, we note that if $\mathcal{E}$ is isomorphic to $R^{s+1}$, then each vector bundle $F_{x}$ is free, and can be endowed, in a natural way, with a basis $\mathcal{B}_{x}$ ([2, p. 985]). Indeed, in this case, $F_{x}$ is a space of multihomogeneous forms as described in $[2,4.3 .13]$, whose basis is formed by the monomials. We will use this basis to identify $F_{x}$ with $R^{N_{x}+1}$ (where $N_{x}:=\operatorname{rk}\left(F_{x}\right)-1$ ).

The following theorem only deals with the trivial vector bundle, i.e. $\mathcal{E}=R^{s+1}$, endowed with the standard Hermitian metric.

Theorem. Let $Z \in Z_{r+1}\left(\mathbf{P}_{R}^{s}\right)$ be a flat, integral, closed subscheme of $\mathbf{P}_{R}^{s}$ of dimension $r+1$ and degree $d(s, d \geq 2, r \geq 0)$, and $n \in\{1, \ldots, d-1\}$ an integer such that $Z_{\bar{K}}$ cannot be written as the union of two closed subschemes of degrees $n$ and $d-n$, respectively. Let $q_{1}, \ldots, q_{v}$ be the distinct prime ideals of $R$ above which the geometric fiber of $Z$ can be written as such a union. Setting $N_{x, r, s}:=\operatorname{rk}\left(\otimes_{i=0}^{r} \operatorname{Sym}^{x}\left(R^{s+1}\right)\right)-1$, we have

$$
\log \prod_{j=1}^{v} N\left(q_{j}\right) \leq \frac{1}{1+\delta_{n, d-n}}\left(\begin{array}{c}
N_{n, r, s}+N_{d-n, r, s} \\
N_{n, r, s}
\end{array}\right) h_{K}(Z)+C(s, d, r, n)
$$

when $h_{K}(Z)$ tends to infinity, where $h_{K}$ is the projective height associated to the standard Hermitian metric on $R^{s+1}$, as defined in [2, 4.1.1] (see also [3, 2.1.5]), $\delta$ is the Kronecker delta function, and $C(s, d, r, n)$ is a function of $s, d, r$, and $n$ that can be given explicitly (see the proof).

Remark. For the hypersurface case $(r=s)$, we find a stricter bound in [4], due to the fact that horizontal hypersurfaces (which correspond to the flat integral closed subschemes here) are (directly) parametrized by a projective space, making it unnecessary to use Chow forms. The $M_{x}$ used there correspond to the $N_{x, r, s}$ for $r=0$ in this paper.

Proof. As noted before, the set $\left\{q_{1}, \ldots, q_{v}\right\}$ is the support of $\pi\left(P_{Z} \cap \mathcal{W}\right)$ in $\operatorname{Spec}(R)$. In particular, $\log \prod N\left(q_{i}\right)=h_{K}\left(\left|P_{Z} \cap \mathcal{W}\right|\right)$. By the arithmetic Bézout theorem $[2,5.5 .1 . i i i]$, we have

$$
\begin{aligned}
h_{K}\left(\left|P_{Z} \cap \mathcal{W}\right|\right) \leq \operatorname{deg}_{K}\left(P_{Z}\right) h_{K}(\mathcal{W})+h_{K}\left(P_{Z}\right) \operatorname{deg}_{K}(\mathcal{W}) \\
+\frac{1}{2}[K: \mathbb{Q}] \operatorname{deg}_{K}\left(P_{Z}\right) \operatorname{deg}_{K}(\mathcal{W})\left(M_{d}+1\right) \log (2) .
\end{aligned}
$$

By definition of $P_{Z}$, its degree equals one. Using the further shortened, and somewhat misleading, notation $N_{x}:=N_{x, r, s}$, we are going to show that the other terms on the right can be bounded as follows:

$$
h_{K}\left(P_{Z}\right) \leq h_{K}(Z)+d[K: \mathbb{Q}]\left(\sigma_{r}+(r+1) \log (s+1)\right),
$$


where $\sigma_{x}=(1 / 2)(x+1) \sum_{m=2}^{x+1}(1 / m)$,

$$
\begin{gathered}
\operatorname{deg}_{K}(\mathcal{W})=\frac{1}{1+\delta_{n, d-n}}\left(\begin{array}{c}
N_{n}+N_{d-n} \\
N_{n}
\end{array}\right), \\
h_{K}(\mathcal{W}) \leq \frac{[K: \mathbb{Q}]}{1+\delta_{n, d-n}} \frac{N_{n}+N_{d-n}+1}{2}\left(\begin{array}{c}
N_{n}+N_{d-n} \\
N_{n}
\end{array}\right) . \\
\log \left((d+1)^{3(r+1)(s+1)} \frac{\left(N_{n}+1\right)\left(N_{d-n}+1\right)}{N_{n}+N_{d-n}+1}\right),
\end{gathered}
$$

leading to the result of the theorem.

Proof of (1). Let $\left\{a_{I}\right\}$ be the coefficients of $P_{Z, K}$ (i.e. of the form $\phi_{\mathbf{1}, Z_{K}}$ ) in the basis $\mathcal{B}_{d}$. Then

$$
h_{K}\left(P_{Z}\right)=\sum_{\sigma} \log \left(\sum\left|a_{I}\right|^{2}\right)^{1 / 2}-\sum_{p} \min _{I} v_{p}\left(a_{I}\right) \log N(p) .
$$

Another height associated to $\mathcal{B}_{d}([2,4.3 .4 .1])$ is

$$
h_{\mathcal{B}}\left(P_{Z}\right):=h_{\mathcal{B}}\left(\mathrm{Ch}_{\mathbf{1}}(Z)\right)=\sum_{\sigma} \log \left(\sum\left|a_{I}\right|\right)-\sum_{p} \min _{I} v_{p}\left(a_{I}\right) \log N(p) .
$$

Clearly, we have $h_{K}\left(P_{Z}\right) \leq h_{\mathcal{B}}\left(P_{Z}\right)$. By [2, Theorem 4.3.8, (4.3.33), and (4.1.2)],

$$
h_{\mathcal{B}}\left(P_{Z}\right) \leq h_{K}(Z)+d[K: \mathbb{Q}]\left(\sigma_{r}+(r+1) \log (s+1)\right) .
$$

In particular, we have $h_{K}\left(P_{Z}\right)=h_{K}(Z)+\mathcal{O}(1)$.

Remark. Before giving the proofs of (2) and (3), let us note that the morphism $\psi$ was used under the notation $\phi_{n}$ in [4], where the degree and height of its image were bounded explicitely. Here we give only sketches of the proofs of (2) and (3), the details can be found in [loc.cit.].

Proof of (2). Let $f_{n}$ (resp. $\left.f_{d-n}\right)$ denote the projection from $\mathbf{P}\left(F_{n}\right) \times \mathbf{P}\left(F_{d-n}\right)$ onto the first (resp. second) coordinate. Using intersection theory, we find

$$
\operatorname{deg}(\psi) \operatorname{deg}_{K}(\mathcal{W})=\operatorname{deg}\left(c_{1} \mathcal{O}_{\mathbf{P}\left(F_{d}\right)}(1)^{N_{n}+N_{d-n}} \cdot\left[\psi_{*}\left(\mathbf{P}\left(F_{n}\right) \times \mathbf{P}\left(F_{d-n}\right)\right)\right]\right),
$$

where $c_{1} \mathcal{O}_{\mathbf{P}\left(F_{d}\right)}(1)$ is the first Chern class of $\mathcal{O}_{\mathbf{P}\left(F_{d}\right)}(1)$. By the projection formula, and the fact that $\psi^{*} \mathcal{O}_{\mathbf{P}\left(F_{d}\right)}(1)=f_{n}^{*} \mathcal{O}_{\mathbf{P}\left(F_{n}\right)}(1) \otimes f_{d-n}^{*} \mathcal{O}_{\mathbf{P}\left(F_{d-n}\right)}(1)$, this implies that

$$
\operatorname{deg}_{K}(\mathcal{W})=\frac{1}{1+\delta_{n, d-n}}\left(\begin{array}{c}
N_{n}+N_{d-n} \\
N_{n}
\end{array}\right)
$$

Proof of (3). As in the proof of (2), we use intersection theory, but this time with metrics. By [2, 4.1.2 and Proposition 2.3.1], we have

$$
\begin{aligned}
h_{K}(\mathcal{W}) & =[K: \mathbb{Q}] \sigma_{N_{n}+N_{d-n}} \operatorname{deg}_{K}(\mathcal{W}) \\
& +\frac{1}{\operatorname{deg}(\psi)} \widehat{\operatorname{deg}}\left(\widehat{c}_{1}\left(\psi^{*} \overline{\mathcal{O}_{\mathbf{P}\left(F_{d}\right)}(1)}\right)^{N_{n}+N_{d-n}+1} \mid \mathbf{P}\left(F_{n}\right) \times \mathbf{P}\left(F_{d-n}\right)\right) .
\end{aligned}
$$


The arithmetic degree on the right is the projective height of $\mathbf{P}\left(F_{n}\right) \times \mathbf{P}\left(F_{d-n}\right)$ associated to the line bundle $\mathcal{L}:=\psi^{*} \mathcal{O}_{\mathbf{P}\left(F_{d}\right)}(1)$ endowed with the pullback $\rho$ under $\psi$ of the standard Hermitian metric on $\mathcal{O}_{\mathbf{P}\left(F_{d}\right)}(1)$. We will bound this height in two steps, using a comparison of metrics on $\mathcal{L}$. First, let $\left(\mathcal{L}, \rho^{\prime}\right)$ denote the line bundle $\mathcal{L}$ endowed with the product metric obtained by taking the standard Hermitian metrics on $\mathcal{O}_{\mathbf{P}\left(F_{n}\right)}(1)$ and $\mathcal{O}_{\mathbf{P}\left(F_{d-n}\right)}(1)$. The associated projective height is

$$
h_{\left(\mathcal{L}, \rho^{\prime}\right)}\left(\mathbf{P}\left(F_{n}\right) \times \mathbf{P}\left(F_{d-n}\right)\right):=\widehat{\operatorname{deg}}\left(\widehat{c}_{1}\left(\mathcal{L}, \rho^{\prime}\right)^{N_{n}+N_{d-n}+1} \mid \mathbf{P}\left(F_{n}\right) \times \mathbf{P}\left(F_{d-n}\right)\right),
$$

which, by the projection formula and the decomposition of the metrized line bundle $\left(\mathcal{L}, \rho^{\prime}\right)$ as a (tensor)product, equals

$$
[K: \mathbb{Q}]\left(\left(\begin{array}{c}
N_{n}+N_{d-n} \\
N_{n}
\end{array}\right) \sigma_{N_{d-n}}+\left(\begin{array}{c}
N_{n}+N_{d-n} \\
N_{n}
\end{array}\right) \sigma_{N_{n}}\right) .
$$

The second step in bounding the height that we want consists of comparing the norms $\|\cdot\|$ and $\|\cdot\|^{\prime}$ associated to $\rho$, resp. $\rho^{\prime}$. Let $\varphi:\left(\mathbf{P}\left(F_{n}\right) \times \mathbf{P}\left(F_{d-n}\right)\right)(\mathbb{C}) \rightarrow \mathbb{R}$ be defined by $\left(\|\cdot\|^{\prime}\right)^{2}=\exp (\varphi)\|\cdot\|^{2}$. For each embedding $\sigma: K \hookrightarrow \mathbb{C}$, and $(a, b)=\left(\left(a_{0}: \ldots: a_{N_{n}}\right),\left(b_{0}: \ldots: b_{N_{d-n}}\right)\right.$ in $\left(\mathbf{P}\left(F_{n}\right) \times \mathbf{P}\left(F_{d-n}\right)\right)_{\sigma}(\mathbb{C})$, we let $f_{a}$, resp. $g_{b}$, be the corresponding multihomogeneous polynomial (in $(r+1)(s+1)$ variables). We have

$$
\exp \left(\varphi_{\sigma}(a, b)\right)=\left(\frac{L_{2}\left(f_{a} g_{b}\right)}{L_{2}\left(f_{a}\right) L_{2}\left(g_{b}\right)}\right)^{2}
$$

where the $L_{2}$-norm $L_{2}(f)$ of a (multi)homogeneous polynomial $f=\sum c_{I} X^{I}$ is $\left(\sum c_{I} \overline{c_{I}}\right)^{1 / 2}$. From results of $[7,3.2]$, we can now deduce that

$$
\sup _{(a, b)}\left(\varphi_{\sigma}(a, b)\right) \leq 3(r+1)(s+1) \log (d+1)
$$

for every $\sigma: K \hookrightarrow \mathbb{C}$. The last step consists of combining this inequality with the results of [2, Proposition 3.2.2] and [1, Lemma 2.6.ii] (see also [4]) to obtain

$$
\begin{aligned}
& h_{(\mathcal{L}, \rho)}\left(\mathbf{P}\left(F_{n}\right) \times \mathbf{P}\left(F_{d-n}\right)\right) \leq h_{\left(\mathcal{L}, \rho^{\prime}\right)}\left(\mathbf{P}\left(F_{n}\right) \times \mathbf{P}\left(F_{d-n}\right)\right) \\
& \quad+[K: \mathbb{Q}] \frac{N_{n}+N_{d-n}+1}{2} \operatorname{deg}(\psi) \operatorname{deg}_{K}(\mathcal{W}) 3(r+1)(s+1) \log (d+1),
\end{aligned}
$$

which, after some simplification, leads to the bound for $h_{K}(\mathcal{W})$ stated in $(3)$.

\section{References}

[1] J.-B. Bost, Intrinsic heights of stable varieties and abelian varieties, Duke Math. J. 82 (1996), 21-70.

[2] J.-B. Bost, H. Gillet, and C. Soulé, Heights of projective varieties and positive Green forms, J. Amer. Math. Soc 7 (1994), 903-1027.

[3] R. Erné, On the degree of integral points of a projective space minus a horizontal hypersurface, prépublication 98-25, Univ. de Rennes I, 1998, submitted to the J. Reine Angew. Math.

[4] R. Erné, Reducibility mod p of hypersurfaces in projective spaces - an application of arithmetic Bézout, prépublication 99-11, Univ. de Rennes I, 1999, revised version (July 1999) accepted by the J. Number Theory. 
[5] A. Grothendieck and J. Dieudonné, Éléments de géométrie algébrique. IV. Étude locale des schémas et des morphismes de schémas, I, Inst. Hautes Études Sci. Publ. Math. 20 (1964), II, Inst. Hautes Études Sci. Publ. Math. 24 (1965), III, Inst. Hautes Études Sci. Publ. Math. 28 (1966), IV, Inst. Hautes Études Sci. Publ. Math. 32 (1967).

[6] J.-P. Jouanolou, Théorèmes de Bertini et applications, Birkhäuser, Boston, 1983.

[7] S. Lang, Fundamentals of diophantine geometry, Springer-Verlag, New York, 1983.

[8] W. Schmidt, Equations over finite fields. An elementary approach, Lecture Notes in Mathematics, 536, Springer-Verlag, Berlin-New York, 1976.

Institut Mathémathiques, Université de Rennes I, Campus De Beaulieu, 35042 Rennes CEDEX, FRANCE

E-mail address: erne@maths.univ-rennes1.fr 